\title{
Research on Design of Hongmu Leftover in Cultural Creative Products
}

\author{
Weilong Gao ${ }^{1}$, Zhuo Tong ${ }^{1}$ and Weixia Gao ${ }^{1 *}$ \\ ${ }^{1}$ School of Art and Textile and Clothing, Changshu Institute of Technology, Changshu, Jiangsu, 215500, China
}

\begin{abstract}
Hongmu is mostly used in furniture. The generation of corner materials in the production process of furniture causes unnecessary waste. Based on the current status of the use of leftover materials, this paper focuses on the feasibility of Hongmu materials in cultural creative design. It puts forward suggestions for the development of leftover materials and cultural creative products to help designers and enterprises use leftover materials more effectively and to maximize the value and cultural connotation.
\end{abstract}

\section{Introduction of Hongmu and Hongmu leftover}

Hongmu is an indispensable carrier of Chinese traditional culture and art. In a broad sense, "Hongmu" refers to various types of hardwood that grow in different places and have different species but are collectively referred to as "Hongmu", as well as traditional Chinese redwood culture and art based on its wood products. Hongmu is a perennial material whose growth rate is slow. Therefore, the condition of the wood is not artificially controlled. The quality of the actual growing wood is basically affected by non-human factors such as the natural environment. The leftover material has the advantages of harder material, high strength, abrasion resistance and good durability. Due to the great quality difference between the sapwood and the heartwood, there are even fewer materials that can be regarded as qualified wood. Therefore, how to maximize the use of leftover materials is particularly important.

Hongmu residue refers to two types of wood. One is the wood that produces defects during the growth process, including knots and decayed materials caused by diseases and insect pests caused by natural environmental factors. It also includes materials due to changes in climate and humidity or harvesting. Cracked materials also include sapwood that does not meet the national standard of Hongmu. The other type refers to qualified wood that is scrapped due to misoperation during machining processing as shown in Figure 1. Due to their different materials, there is a huge disparity in the prices of Hongmu. The thesis discusses the redesign and practical application of leftover materials. Many Hongmu resources are gradually depleted, and it is difficult to meet the needs of the expanding Hongmu market. Under the premise of emphasizing a low-carbon economy and advocating green environmental protection, if the Hongmu can be reused and further innovative design, the resources can be fully utilized, which can create greater value and benefits, and make more people feel the charm of Hongmu culture and art[1].

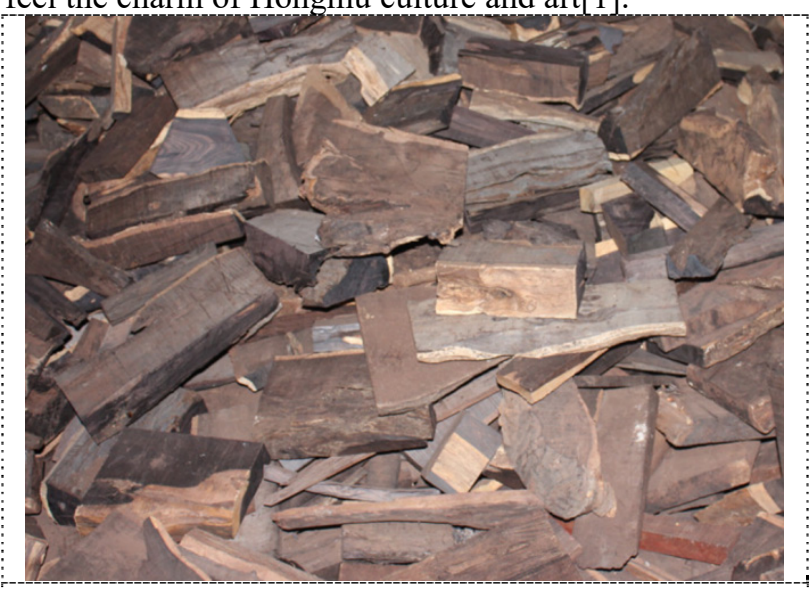

Figure 1. Hongmu scraps in the factory

\section{The Status of Hongmu cultural creative products industry}

Based on the country's support for cultural and creative industries and the improvement of people's living standards, the domestic creative industry is booming, and many types of cultural products are emerging endlessly, not only sought after by young people, but also become the main content of business exchanges, gift gifts, and collections. Among these creative products, wood products have become a unique and attractive creative scenery line with their special and beautiful texture. They are unique and stand out from other products, showing objective market prospects and commercial value. In the field of wooden creative cultural products, the development of the category of Hongmu cultural creative products is relatively lagging, as shown in the following aspects[2-3]. 


\subsection{Limited market development}

No matter from the perspective of consumers or professionals, the market development of Hongmu leftover cultural and creative products is not comprehensive enough. At present, the output of Hongmu creative products invested in the market exceeds supply. This is not because the consumer's purchasing power is not good, but because Hongmu is relatively mature due to its material characteristics, stable temperament, and product design is not modern enough, so it is less popular with young people and the range of creative product audiences is limited. On the other hand, many products themselves are not sophisticated enough, and the flood of products the Hongmu cultural and creative product market.

\subsection{Lack of creativity}

At present, the design sense of Hongmu creative products is not strong, the shape is single, and the types and classification boundaries of products are vague. There are many kinds of Hongmu, but after the creative products are made, the classification of Hongmu is weakened. Most of the products are out of date, and their functions have not been fully developed. Most of them are limited to stationery, which is not related to daily life.

\subsection{Insufficient connection between design and production}

The connection between the design enterprise and the factory is not enough. According to the waste degree of the Hongmu factory, the factory does not classify the Hongmu materials reasonably. The design enterprise only designs the style unilaterally, does not have a deep understanding of the Hongmu leftover material. To some extent, it's "An Armchair Strategist". These two aspects do not have the very good fusion progress. Designers need to fully control the remaining materials in order to make good use of Hongmu leftover materials and increase utilization.

\section{Suggestions on the development of Hongmu leftover cultural and creative products}

\subsection{Classification of materials}

Hongmu leftovers are not the same as bad quality wood. The focus of their reuse lies in resource conservation and integration and reuse. If the quality is not high enough, the creative products can only be crude, which cannot show the Hongmu characteristics and cultural atmosphere. Therefore, when selecting Hongmu residues, we must also control the quality. The residues are effectively classified and screened before selection.

\subsection{Diverse development design}

The variety of Hongmu creative materials is diversified. Diversity here refers not only to the diversified appearance but also the combination of materials. Hongmu can try to combine with many materials such as ceramics, precious metals, agate and so on. Hongmu embodies the calm and heavy natural gas quality. Adding other texture materials to it can integrate multiple sensations, such as adding ceramics to soften the hardness and weight of wood. In addition to the combination of different materials, due to the small size of the remaining material, the designer can completely propose the design of the superposition of Hongmu leftover materials. Although its single volume is not suitable for many large-volume designs, "stacking" makes the design break the remaining material.

\subsection{Emotional design}

Hongmu leftover creative products are emotional. In this era, design itself is no longer a simple function. A product is more fancy than a comprehensive reflection of the connotation of life as a subject. Only emotional things have the soul and the possibility to communicate with others. When the product has its own personality, it will attract the consumer group. This group is led by the product's personality. The Hongmu leftover material creative product must be able to convey its emotional characteristics and fully express the design concept and soul. Inspiring creative products is the reason why consumers buy. The high pressure and fast pace of today's society have made life more and more mechanical, and have lost some of the original things. Jimu Industrial Design Co., Ltd. designed wooden dolls for the purpose of communicating back to childhood. Starting from the beautiful and short childhood memories treasured by users, it was based on tide, cuteness, and unique product styles. It used Hongmu leftover materials and other materials, showing a unique product texture, as shown in Figure 2.

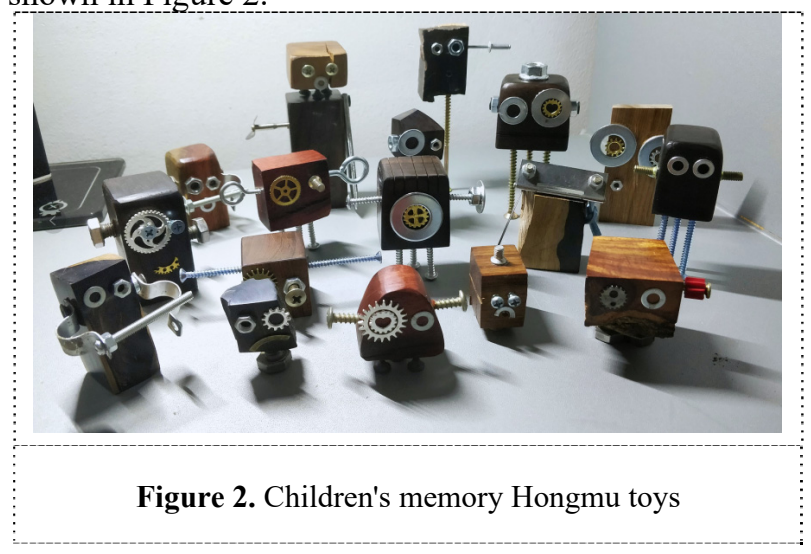

\subsection{User experience}

Since the Premier of the State Council proposed the concept of Internet Plus at the two sessions, it has quickly become popular and has been favored by many enterprises. The development of Hongmu leftover 
materials is undoubtedly also affected by Internet Plus. However, in the era of the experience economy, Hongmu leftover cultural and creative product while development Internet plus must enhance user experience to let users participate in the formation or production process of products and create unique objects. The redesign of the Hongmu leftover material itself can gather the creative ideas of consumers, allowing users to experience the unique culture of Hongmu in their own creativity and production. This is the most direct and unique customized service, making the product experience more emotional and personal. For example, students use their university campus culture as an example to develop a series of Hongmu cultural and creative products, as shown in Figure 3.

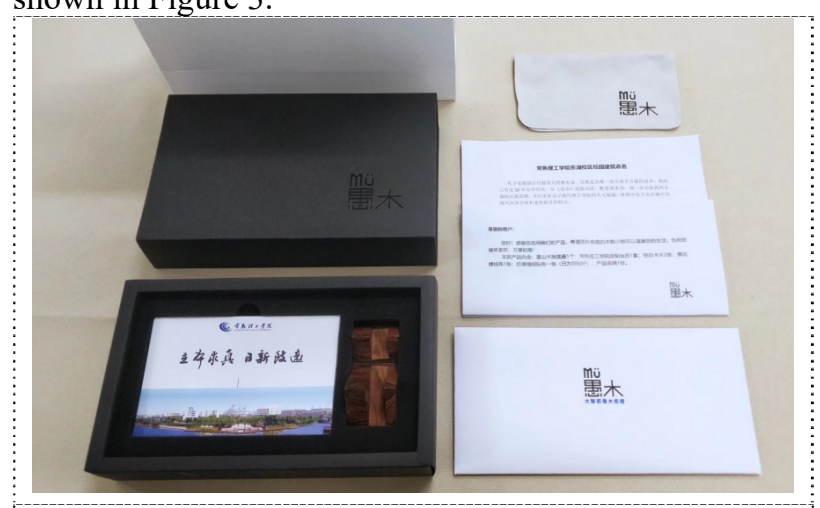

Figure 3. Hongmu campus cultural calendar

\section{Summary}

The cultural and creative design of Hongmu leftover materials can not only achieve the sustainable development of resources, but also carry forward the traditional Chinese culture to let ordinary people feel the charm of Hongmu. Of course, there is still a long way to go for the market development of Hongmu leftovers cultural products. From a product to a product series to a brand, the corresponding design methods, follow-up design techniques and other development ideas are different. In the design process of using Hongmu leftovers, different design techniques need to be mastered, and suitable creative ideas should be adopted to exert its maximum role and energy under different environments.

\section{Acknowledgments}

This research was financially supported by Research on Green Design of Hongmu Furniture Leftovers (Grant NO. XJDC2019306), Research Project of Changshu Institute of Technology (Grant NO. KYZ2017103Z).

\section{References}

1. Xuan Y, Zhou C.M, Fu X.M. (2015) Discussion on the development of cultural and creative products using Hongmu residual materials. Furniture, 6: 99103.
2. Song S.L, Zhong S.L. (2015) Redesign of Hongmu remnants.Design, 5: 103-104.

3. Zhang H.W,Liu Y.P.(2016) Discussion on the development of cultural and creative products using Hongmu furniture residual materials. Modern decoration (theory), 8: 46.

4. Zhang H.X, Li J. (2019) Modern design based on Hongmu residue. Art research, 6: 19-21. 